\title{
Close Conjunction Detection on Parallel Computer
}

\author{
Liam M. Healy \\ Naval Research Laboratory, Washington, D.C. 20375-5355
}

\begin{abstract}
Close conjunction detection is the task of finding which satellites will come within a given distance of other satellites. The algorithms described here are implemented on the Connection Machine (CM) in a program called CM-COMBO. It will find close conjunctions of satellites over a time range for one, a few, or all satellites against the original or another catalog and works with an arbitrary propagator. The problem of comparing an entire catalog against itself is beyond the computing power of current serial machines. This program does not prefilter any orbits and does not make any assumptions about the type of orbit (that it be nearly circular, for instance). This paper describes the algorithm for this computation, the implementation on the $\mathrm{CM}$, and results of several studies using this program.
\end{abstract}

\section{Introduction}

A CLOSE conjunction determination algorithm is one that takes a catalog of satellites and determines which satellites come within some specified distance of another over a period of time. Such an algorithm should work with any propagator and make as few assumptions as possible about the types of orbits of satellites in the catalog. A propagator computes the phase space points at some time step after the initial time, given the phase space values at an initial time. The algorithm described here does not depend on any particular propagator.

Traditionally, a single satellite is compared against a catalog of satellites to find close encounters. This is the function of a program called COMBO (Calculation of Miss Distance Between Objects) running on serial computers at U.S. space surveillance centers. More challenging, however, is the problem of the comparison of a large number of satellites, comparisons that are either all to all within a given catalog or between two large sets of satellites. This capability would be useful, for example, in the study of debris, the hazard to a proposed constellation of satellites, or the danger due to overcrowding of the orbital environment. By taking advantage of parallel processing on a computer such as the Connection Machine (CM), it is feasible to do this.

The algorithms described here have all been implemented in $\mathrm{CM}$ Fortran on a CM-5E with 256 nodes.

\section{Single Satellite}

For comparison of a catalog against a single satellite at a particular time, a parallel processor is a natural. With the Cartesian coordinates of the primary satellite available to all processors and the Cartesian coordinates of all the other satellites at the other processors, a directdistance computation may be performed in parallel. This method as described is adequate only for finding close pairs at a particular time and not for pairs that come briefly within the desired critical distance and then pass out in the time interval.

In order to find close conjunctions in a time interval, we do a Taylor series extrapolation on the motion. We consider at one time step a continuous time range, say an interval of time $T$ centered on the time $t_{0}$. Thus, the propagator would compute positions and momenta at time $t_{0}$ and take a step to compute them at a time $T$ later, and so on. We wish to find all close conjunctions in the range $t \in\left[t_{0}-T / 2, t_{0}+T / 2\right]$. With the velocity information that comes back from the propagator, we can extrapolate to find the closest approach. Approximating the relative displacement vector of the two satellites with a Taylor series

$$
x=x_{0}+\left(t-t_{0}\right) \dot{x}_{0}+\frac{1}{2}\left(t-t_{0}\right)^{2} \ddot{x}_{0}+\frac{1}{6}\left(t-t_{0}\right)^{3} x_{0}^{(3)}+\cdots
$$

we may find the point of closest approach by the minimum value of $\boldsymbol{x} \cdot \boldsymbol{x}$. Truncating Eq. (1) at the linear term, we have

$$
x \cdot x=x_{0} \cdot x_{0}+2\left(t-t_{0}\right) x_{0} \cdot \dot{x}_{0}+\left(t-t_{0}\right)^{2} \dot{x}_{0} \cdot \dot{x}_{0}
$$

The time at which this is an extremum can be solved by finding the root of the derivative,

$$
\frac{\mathrm{d}}{\mathrm{d} t} x \cdot x=2 x_{0} \cdot \dot{x}_{0}+2\left(t-t_{0}\right) \dot{x}_{0} \cdot \dot{x}_{0}=0
$$

To lowest order, the time of closest approach occurs at

$$
t_{\text {closest }}=t_{0}-\frac{x_{0} \cdot \dot{x}_{0}}{\dot{x}_{0} \cdot \dot{x}_{0}}
$$

The vector $\boldsymbol{x}_{0}$ and its derivative $\dot{\boldsymbol{x}}_{0}$ are usually calculated by the propagator. The distance at the time of closest approach is easily calculated from this time, the initial values, and the Taylor series (1).

After all the estimated closest approach distances and times have been computed and likely close conjunctions within the time band extracted, they may be refined using a Newton method iteration in parallel using the actual propagator. Thus, the final answer is available to any desired accuracy within the capabilities of the specified propagator. Although computing $\boldsymbol{x} \cdot \boldsymbol{x}$ with more terms in the Taylor series (1) and solving for the time of closest approach by reversion

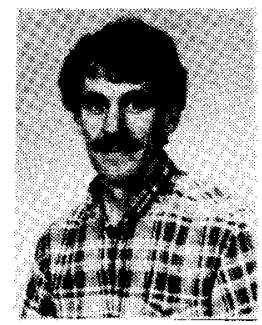

Liam M. Healy received an A. B. in physics from Harvard in 1979, an M.S. in 1981 and Ph.D. in 1986, both from the University of Maryland, College Park, in Physics. His Ph.D. dissertation was on beam dynamics in particle accelerators. Since 1987 he has been at the Naval Research Laboratory, first as a NRC Cooperative Research Associate and currently as a Research Physicist in the Mathematics and Orbit Dynamics section of the Naval Center for Space Technology. His research interests are in orbit dynamics and Hamiltonian systems, particularly using symbolic and parallel computation.

Received Feb. 23, 1994; revision received Oct. 4, 1994; accepted for publication Nov. 28, 1994. This paper is declared a work of the U.S. Government and is not subject to copyright protection in the United States. 
of series give more accurate results, this turns out to be an ineffective use of computer time. For step sizes of 2 min the error made by this linear approximation on the actual catalog is typically about $10 \mathrm{~ms}$ on average, resulting in an average distance error of about $2 \mathrm{~m}$ (the reason that the distance of closest approach changes so little relative to the change in time is that the distance-time curve is usually quite flat near the closest approach). The addition of higher orders in the Taylor series, although improving these figures, greatly slows the program. Since the final answer will be refined with the actual propagator anyway, there is little point in doing the extra computations earlier.

Aside from being designed for a parallel computer, this algorithm has advantages over others previously reported. Hoots et al. ${ }^{1}$ describe a method that uses extensive prefiltering of orbits, designed to remove from consideration those satellites that could never be near the primary. This requires careful consideration of different kinds of orbits and detailed knowledge of the perturbations to be considered. Furthermore, they use a Newton method iteration with an idealized motion model to solve for the point of closest approach for all satellite pairs not removed by the prefilter; the final stages of iteration use the full propagator. Prefiltering the set requires extra analysis and programming; furthermore, it is of very little advantage in a parallel computer. Mietz ${ }^{2}$ also uses prefiltering, which he calls a "discard phase." Alfano and Negron ${ }^{3}$ use a curve-fitting approach, which requires values at multiple time steps. They use a set of four points at once, blending two parabolas formed from the first three and last three points. The blending process is repeated with successive sets of points through the whole data set. The method presented here is simpler conceptually and computationally and requires only positions and velocities at a particular time in order to determine conjunctions over an interval. Dybczyński et al. ${ }^{4}$ attempt to solve the problem of the minimum distance between two orbits, rather than two orbiting satellites. Their method is iterative and is based on strictly Keplerian orbits. So too is the method of Beerer and Bauer. ${ }^{5}$ Theirs is based on spherical trigonometric relationships and is valid only for circular orbits.

None of the previous work mentioned attempts to handle the problem of an all-to-all comparison, only of a particular (primary) satellite against the catalog. For a small number of primaries, one can compute in succession using this one-to-all comparison, but with too many primaries, a different approach is required.

\section{Close Conjunction Sieve}

The previous section has shown how to compute close conjunctions between a primary (or limited number of primaries) and the remaining catalog of satellites. Of different interest, for example, in verifying debris hazard models, is the hazard of any satellite to any other. In this case, specific conjunction detection is the same, but the logistics of comparison will be different. To make matters easier, consider first the conjunction determination at a particular time.

The computation of close miss distances involves finding all pairs of satellites within a set that are within some specified critical distance $c$ at a given time. The general approach is as follows. One may take the Cartesian coordinates $x=[x, y, z]^{T}$, in arrays stored on the parallel computer and duplicate the set:

$$
\begin{aligned}
& \begin{array}{|l|l|l|l|}
\hline x_{1} & x_{2} & \cdots & x_{n} \\
\hline x_{1} & x_{2} & \cdots & x_{n} \\
\hline
\end{array}
\end{aligned}
$$

Then, with one copy fixed, we shift all three arrays by one and compute in parallel the distance of aligned coordinates,

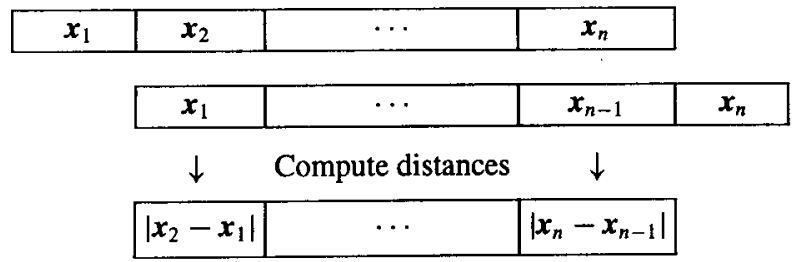

saving in a separate array pairs that are closer than some critical distance $c$. Then we repeat the shift-distance-save operation until all possible pairs have been compared. Counting the displacement of the upper array to the left relative to the lower array by $s$, we compare $\left|x_{i+s}-x_{i}\right|$ in parallel for all $1 \leq i \leq n-1$ and serially $1 \leq s \leq n-1$.

This method will determine all close pairs at the time, but it is unnecessarily time consuming. We may greatly reduce the computations by sorting the elements in advance. The position vectors are rearranged so that one Cartesian coordinate of the vector $x$, say $z$, is sorted in ascending order. First observe that if two satellites differ by more than $c$ in one coordinate, they are more than $c$ apart:

$$
\left|z_{i}-z_{j}\right|>c \Rightarrow\left|x_{i}-x_{j}\right|>c
$$

As a consequence of the sorted coordinates, if two satellites in the array differ by more than $c$ in one coordinate, then a pair formed by the first satellite and another later in the array than the second will also differ by more than $c$ in the coordinate:

$$
k>j>i \text { and }\left|z_{j}-z_{i}\right|>c \Rightarrow\left|z_{k}-z_{i}\right|>c
$$

These together imply that if, at a certain stage of the computation, the minimum of the differences of the $z$ values is greater than $c$, no further shifts will produce an aligned pair whose Cartesian distance is less than $c$. Thus we modify the algorithm above; if we let $p$ be

\begin{tabular}{|c|c|c|c|c|}
\hline$x_{p^{-1}(1)}$ & $x_{p^{-1}(2)}$ & $\cdots$ & $\boldsymbol{x}_{p^{-1}(n)}$ & \\
\hline & $\boldsymbol{x}_{p^{-1}(1)}$ & $\cdots$ & $x_{p^{-1}(n-1)}$ & $x_{p^{-1}(n)}$ \\
\hline & $\downarrow$ & $\begin{array}{l}\text { Compute } \\
\text { distances }\end{array}$ & $\downarrow$ & \\
\hline & $\begin{array}{l}\mid x_{p^{-1}(2)} \\
\quad-x_{p^{-1}(1)} \mid\end{array}$ & $\ldots$ & $\begin{array}{l}\mid x_{p^{-1}(n)} \\
\quad-x_{p^{-1}(n-1)} \mid\end{array}$ & \\
\hline
\end{tabular}
the ranking of satellites by the $z$ value, i.e.,

$$
z_{p^{-1}(1)} \leq z_{p^{-1}(2)} \leq \cdots \leq z_{p^{-1}(n)}
$$

shifting and finding distances produce

Now, however, after each shift an extra step checks the minimum $z$ differences,

$$
\min _{1 \leq i \leq n-s}\left|z_{p^{-1}(i+s)}-z_{p^{-1}(i)}\right|
$$

for shift $s$ in parallel of all aligned pairs. If this minimum is greater than $c$, we terminate the computation, confident that all pairs that are within a distance $c$ of each other have been found. Since the need for most of the shifts, which are done sequentially, is eliminated, sorting improves the speed of the close conjunction determination by a factor of more than 20 .

Having found a fast way to determine all conjunctions within a catalog at a particular time, we seek to expand this to the case of the continuous-time interval $t \in\left[t_{0}-T / 2, t_{0}+T / 2\right]$ using the method described in Sec. II. We shall need to modify the rearrangement of the arrays, however. If we sort merely by the value at a particular time and then shift until the minimum difference of the $z$ values is at least $c$, we might miss a pair that is, at $t_{0}$, greater than $c$ apart but will (or had) approached to less than $c$ within the time interval. Thus we choose a different arrangement: The two arrays that are to be compared will not have the same ordering.

For each satellite, we determine the lowest $z$ value and the highest $z$ value in the time interval, i.e.,

$$
\begin{aligned}
& z^{\max }=\max _{t \in\left\{t_{0}-T / 2, t_{0}+T / 2\right]} z(t) \\
& z^{\min }=\min _{t \in\left[t_{0}-T / 2,\{\}+T / 2\right]} z(t)
\end{aligned}
$$

These are fairly easy to determine. If the sign of the $z$ velocity is the same at the beginning and end of the time interval and the interval is sufficiently small, there is no turning during the interval. Depending on this sign, the $z$ at each end of the time interval is the minimum 
or maximum. If the signs are different, there is a turning. One can use the time derivative of the $z$ component of the Taylor expansion (1) to solve for the time of $z$ turning and the extremal distance.

The arrays of Cartesian positions at $t_{0}$ shifted left will be arranged so that the corresponding values $z^{\mathrm{min}}$ would be arranged in increasing order, and the other array will be arranged so that the values $z^{\max }$ would be arranged in increasing order. Note that the two orderings have no relation to one another: A satellite with $z^{\text {min }}$ at position $i$ does not necessarily have $z^{\max }$ at position $i$ too. If $l$ is the ranking of satellites by $z^{\min }$ and $h$ the ranking by $z^{\max }$, then our comparison looks like, for an example case of $s=1$,

\begin{tabular}{|c|c|c|c|c|}
\hline$x_{t^{-1}(1)}(t)$ & $x_{l^{-1}(2)}(t)$ & $\cdots$ & $x_{l^{-1}(n)}(t)$ & \\
\hline & $x_{h^{-1}(1)}(t)$ & $\cdots$ & $x_{h^{-1}(n-1)}(t)$ & $x_{h^{-1}(n)}(t)$ \\
\hline & $\downarrow$ & $\begin{array}{c}\text { Estimate } \\
\text { minimum } \\
\text { distances } \\
\text { over interval }\end{array}$ & $\downarrow$ & \\
\hline & $\begin{array}{l}\mid x_{l^{-1}(2)}(t) \\
\quad-x_{h^{-1}(1)}(t) \mid\end{array}$ & $\cdots$ & $\begin{array}{l}\mid x_{l^{-1}(n)}(t) \\
\quad-x_{h^{-1}(n-1)}(t) \mid\end{array}$ & \\
\hline
\end{tabular}

Here, $t \in\left[t_{0}-T / 2, t_{0}+T / 2\right]$ lies in the time interval and is the estimated time at which the two satellites are closest and is in general different for each pair.

In the single-time method, the arrays were first aligned and then shifted once before the start of the comparison; then the comparison proceeded with $s=1,2, \ldots, s_{\max }$. This ensured that no duplicate pairs and no self-pairs (both members of the same satellite) were compared. Here, since the orderings are different, we have no such assurance. We do not necessarily start at $s=1$ but at a value $s_{\min }$ yet to be found, which will in general be less than zero; that is, the upper array starts out displaced to the right of the lower array.

The maximum value of the shift $s$ is determined in the same way as the fixed-time case described above. When the minimum of the $z$ differences between the two arrays is greater than the critical distance, we can be assured that this alignment or further shifts left will not produce any new close pairs. Thus if

$$
\min _{i}\left[z_{l^{-1}(i+s)}^{\min }-z_{h^{-1}(i)}^{\max }\right]<c
$$

no further shifting is necessary. Note that this time we do not take an absolute value: It is the $z^{\max }$ subtracted from the $z^{\min }$. For $z^{\max }>$ $z^{\text {min }}$, this number is negative and thus is always less than the critical distance, which must be nonnegative. Only when $z^{\max }<z^{\min }$ for all aligned pairs is it possible to consider an end to shifting.

The minimum value of the shift $s$ is determined in a completely different manner. The shift needed to align satellite $i$ with satellite $j$ will be the lesser of $\Xi_{i j}$ and $\Xi_{j i}$, where $\Xi_{i j}=h(i)-l(j)$, keeping in mind that a given satellite pair can be matched twice $\left(z^{\mathrm{min}}\right.$-ordered to $z^{\max }$-ordered and $z^{\max }$-ordered to $z^{\min }$-ordered). Let us call the rank difference $\Xi_{i}$ for a satellite $i$ the difference between the high and low ranking,

$$
\Xi_{i} \stackrel{\text { def }}{=} \Xi_{i i}=h(i)-l(i)
$$

One of the following must be true for all $i$ and $j, i \neq j$ :

$$
\begin{aligned}
& h(j)<h(i) \Rightarrow h(j)-l(i)<h(i)-l(i) \Rightarrow \Xi_{j i}<\Xi_{i} \\
& h(i)<h(j) \Rightarrow h(i)-l(j)<h(j)-l(j) \Rightarrow \Xi_{i j}<\Xi_{j}
\end{aligned}
$$

Thus $\min \left[\Xi_{i j}, \Xi_{j i}\right]<\max \left[\Xi_{i}, \Xi_{j}\right]$. To cover all possible pairs, we need only a negative shift given by

$$
\max _{i \neq j}\left[\min \left[\Xi_{i j}, \Xi_{j i}\right]\right]<\max \left[\Xi_{i}\right]
$$

where $\max \left[\Xi_{i}\right]$ is the largest rank difference of all the satellites. The most negative shift therefore is $s(i)=1-\max \left[\Xi_{i}\right]$.
Intuitively, the satellites with the highest rank difference are the fast movers in the $z$ direction: They have the largest difference between minimum and maximum $z$. More accurately, they are the satellites that cross the most number of other satellites in the $z$ direction during the time interval. Notice, however, that the algorithm makes no assumptions about how satellites move or what their orbits are.

This treats only the case of conjunction determination all-to-all within one set. It is possible by simple extension to make a comparison of two large sets. By keeping a logical array, we can divide a single set of positions into two logical sets. A conjunction of satellites belonging to the same set can be rejected, so that only conjunctions between the sets are kept. An application might be the comparison of the existing orbital catalog with a hypothetical constellation or a simulated debris cloud.

\section{Direct Comparison}

While the method described above will work to compare two large sets of satellites, or all to all within one set, we can greatly improve performance with a conceptually simple idea. We can reduce the number of shifts by putting those satellites with the highest rank difference and those with which they would be compared into a direct comparison.

With actual catalogs, the value $s(i)$ can be quite negative, necessitating many serial shifts. To cut computation time, remove the fastest movers and do a direct comparison. Say that satellite $i=i_{1}$ has the maximum value of rank difference, $\Xi_{i_{1}}=201$, but that the next highest satellite $i=i_{2}$ has $\Xi_{i_{2}}=101$. Then, by directly comparing satellite $i_{1}$ 's position with the 100 satellites it would have been shifted over, we can start at shift $s_{\text {start }}=-100$ instead of $s_{\text {start }}=s\left(i_{1}\right)=-200$. This speeds things up tremendously because the direct comparison can be done in parallel, whereas the shifts are done one after another. In fact, several of the top few satellites in rank difference can be brought out specially for a direct comparison in parallel, greatly cutting the number of shifts.

Assume the rank differences have been sorted in decreasing order, so that $\alpha$ is the index of the satellite with the highest rank difference in the original array and $\beta$ the next. Now look at where $\alpha$ occurs in the $z^{\min }$ - and $z^{\max }$-sorted arrays:

$$
\begin{array}{|l|l|l|l|l|l|l|l|l|}
\hline \cdots & \boldsymbol{x}_{a} & \boldsymbol{x}_{\boldsymbol{b}} & \boldsymbol{x}_{\boldsymbol{c}} & \cdots & & \boldsymbol{x}_{\boldsymbol{\alpha}} & \cdots & z^{\mathrm{min}} \text {-sort } \\
\hline
\end{array}
$$

$$
\begin{array}{|l|l|l|l|l|l|l|}
\hline \cdots & \boldsymbol{x}_{\alpha} & \cdots & \boldsymbol{x}_{d} & \boldsymbol{x}_{\boldsymbol{e}} & \boldsymbol{x}_{f} & \cdots \\
\hline
\end{array} z^{\text {max }} \text {-sort }
$$

Assuming this diagram shows the alignment with $s=s(i)$, we see that the satellite $\alpha$ will be compared with two other satellites, labeled $a$ and $f$. At the next shift, $s=s(i)+1$, it will be compared with the satellites labeled $b$ and $e$. At the third shift, it will be compared with the satellites labeled $c$ and $d$. To alleviate the need for the three sequential comparisons and shifts, we may transfer these values to separate arrays for direct comparison:

$$
\begin{aligned}
& \begin{array}{|l|l|l|l|l|l|l|}
\hline \boldsymbol{x}_{\alpha} & \boldsymbol{x}_{\alpha} & \boldsymbol{x}_{\alpha} & \boldsymbol{x}_{\alpha} & \boldsymbol{x}_{\alpha} & \boldsymbol{x}_{\alpha} & \cdots \\
\hline
\end{array} \\
& \begin{array}{|l|l|l|l|l|l|l|}
\hline \underbrace{\boldsymbol{x}_{a}}_{L} & \boldsymbol{x}_{b} & \boldsymbol{x}_{c} & \boldsymbol{x}_{\boldsymbol{x}_{d}} & \boldsymbol{x}_{\boldsymbol{e}} & \boldsymbol{x}_{f} & \cdots \\
\underbrace{}_{H}
\end{array}
\end{aligned}
$$

Assuming that $\Xi_{\beta} \leq \Xi_{\alpha}-3$, we may now start with initial shift $t=s(\alpha)+3$ and not lose anything. The new array that gets the high movers will be called the broadcast (B) array, and the new array to which they are compared will be called the comparee (C) array. The size of these arrays I will call BC array size. In the diagram, the letter $\mathbf{L}$ designates replaced comparisons of the broadcast satellite with the $z^{\text {min }}$-ordered array, and $\mathrm{H}$ designates replaced comparisons with the $z^{\max }$-ordered array.

Depending on the size of the broadcast and comparee array, one may pick the highest $n$ satellites in rank difference and put them with their comparee satellites into these arrays. Each satellite requires a minimum shift of $s(i)=1-\Xi_{i}$. For each satellite, the number of potential conjunctions lost from increasing the initial shift to $t$ [less negative than $s(i)$ ] is $2(t-s(i))=2\left(\Xi_{i}+t-1\right)$, the factor of 2 arising from the $L$ and the $H$ comparison. Table 1 shows the arrangement of the $\mathrm{B}$ and $\mathrm{C}$ arrays, giving, for each element, the 
Table 1 Arrangement of arrays for direct comparison

\begin{tabular}{lccc}
\hline \hline Index & B array & C array & Comment \\
\hline 1 & $\alpha$ & $a=l^{-1}[h(\alpha)+s(\alpha)]$ & Start $\alpha \mathrm{L}$ \\
2 & $\alpha$ & $b=l^{-1}[h(\alpha)+s(\alpha)+1]$ & \\
3 & $\alpha$ & $c=l^{-1}[h(\alpha)+s(\alpha)+2]$ & \\
$\vdots$ & $\vdots$ & $\vdots$ & \\
$t-s(\alpha)$ & $\alpha$ & $l^{-1}[h(\alpha)+t-1]$ & End $\alpha \mathrm{L}$ \\
$1+t-s(\alpha)$ & $\alpha$ & $f=h^{-1}[l(\alpha)-s(\alpha)]$ & Start $\alpha \mathrm{H}$ \\
$2+t-s(\alpha)$ & $\alpha$ & $e=h^{-1}[l(\alpha)-s(\alpha)-1]$ & \\
$3+t-s(\alpha)$ & $\alpha$ & $d=h^{-1}[l(\alpha)-s(\alpha)-2]$ & \\
$\vdots$ & $\vdots$ & $\vdots$ & End $\alpha \mathrm{H}$ \\
$2[t-s(\alpha)]$ & $\alpha$ & $h^{-1}[l(\alpha)-t+1]$ & Start $\beta$ L \\
$1+2[t-s(\alpha)]$ & $\beta$ & $l^{-1}[h(\beta)+s(\beta)]$ & \\
$2+2[t-s(\alpha)]$ & $\beta$ & $l^{-1}[h(\beta)+s(\beta)+1]$ & \\
$\vdots$ & $\vdots$ & $\vdots$ & \\
\hline \hline
\end{tabular}

Note: Index is index in two arrays, $\mathrm{B}$ array is index in original order of satellite in $\mathrm{B}$ array, and $\mathrm{C}$ array is index in original order of satellite in $\mathrm{C}$ array; $\alpha$ and $\beta$ are original ordering indices of high movers, and $a \ldots, f$ correspond to example in diagrams.

index in the original array of the Cartesian coordinates that constitute that element. The comments indicate where each block begins and ends. The ordering of the satellites with high rank difference, i.e., what is $\alpha, \beta$, etc., is not important. One might sort the rank differences and select $\alpha, \beta, \ldots$, but it is more convenient and faster to pick a value of $t$, compute the total number of direct comparisons necessary, see if they will fit in the arrays, and adjust $t$ if necessary. Then the satellites with high rank difference may be picked off in the order they occur in the original array.

\section{Categorizing Conjunctions}

There are essentially two types of conjunctions observed in the catalog of Earth-orbiting satellites: coincidental conjunctions and traveling partners. The former are satellites that once or periodically are within the critical distance, because they are in different orbits. The latter are satellites that, by accident or by design, are orbiting together, such that they are always or nearly always within the critical distance of one another. A prime example of this is the Russian space station Mir, which is recorded in the U.S. Space Command catalog as seven separate pieces. Clearly, the coincidental conjunctions are of primary interest; without assuming prior knowledge of traveling partners in a given catalog, we would like to sort the conjunctions into the two classes. The traveling partners, although for the most part actually known about in advance, are not assumed to be given. Instead, there are multiple cues that can help determine the type of conjunction for a pair.

At each time step, there are two computations made to identify conjunctions. First, the distances at the current time $t_{0}$ are computed. Second, the time and distance of closest approach are estimated according to Eq. (4). If the first distance is less than the critical distance $c$, or if the second distance is less than $c$ and the time within one-half the interval, $T / 2$ of $t_{0}$, then these values are saved along with the identifying numbers for each member of the pair and a key composed from these identifiers that uniquely identifies the pair. At each step, this information is sorted by key and duplicate conjunctions, which are now adjacent in the array, removed.

After the analysis at a particular time interval is done and the information saved, the propagator advances to the next time step and the process is repeated. After the entire period of simulation has been completed, there will be a large collection of conjunction information from all the time steps. To make sense of this, we will need to sort and analyze it.

The global analysis of conjunction information must be able to make sense of different kinds of distance functions in a way that would match a knowledgeable person's judgment. Consider the four different distance functions given in Fig. 1; in each of these plots the dashed line indicates the critical distance. Plot a is clearly a coincidental conjunction, and there should be no problem categorizing it as such. Likewise, plot $b$ is clearly a traveling partner. Plot $c$ shows the pitfall of judging the two cases based on a hard cutoff $c$ : It is obviously just like Fig. 1b, but at a greater distance, so that part

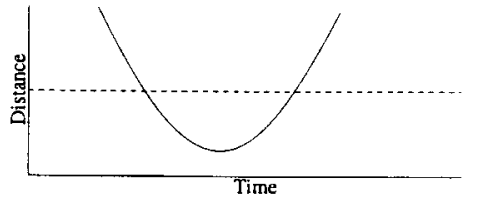

a) Coincidental conjunction

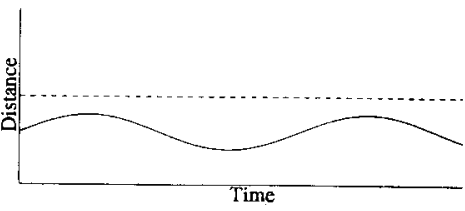

b) Traveling partners

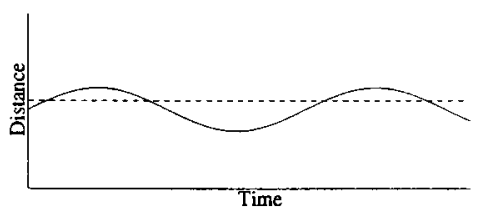

c) Potentially ambiguous case

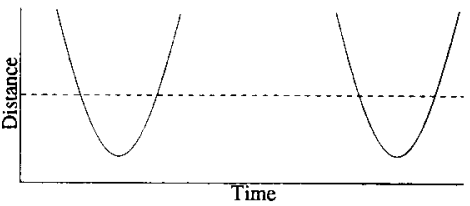

d) Two separate coincidental conjunctions

Fig. 1 Some idealized distance functions for satellite pairs; dotted lines indicate critical distance $c$.

of the time the two satellites are further than $c$ apart. On the other extreme, however, as the far distance increases, it becomes like plot $\mathrm{d}$, which we would want to categorize as two separate coincidental conjunctions. Identifying criteria to accurately discriminate between these cases is difficult; in practice those given below when applied to real catalogs and a realistic critical distance $c$ rarely produce misclassifications.

To formally classify the conjunctions, we sort the conjunction information from all times using the pair key. This information includes the type of conjunction: distance within $c$ at $t_{0}$ (a current contact) or estimated within the time interval (an estimated contact) or both. If at least half the time steps have a conjunction of a particular pair and at least $90 \%$ of these are a current contact, that pair is considered a traveling partner. Any pair that is not a traveling partner and has an estimated contact is a coincidental conjunction or conjunctions.

A third possibility must be considered, that of two (or more) separate coincidental conjunctions involving the same pair of satellites, which might otherwise be interpreted as traveling partners, such as Fig. Id. If there is more than one estimated contact, and the standard deviation of the times of the multiple approaches is more than some threshold, say $2.0 \mathrm{~s}$, we shall take it as multiple coincidental conjunctions. Any newly identified multiple coincidental conjunctions are marked with each conjunction separate, and analysis is then redone as two separate coincidental conjunctions.

For the traveling partner, we may compute the mean and standard deviation of the distance and the percentage of time steps in which the distance is within the critical distance. For the coincidental conjunctions, we may find a more precise time and distance of closest approach using a Newton search procedure and the actual propagator (rather than an extrapolation). This is not very computationally intensive, because all coincidental conjunctions obtained through the whole run are refined at the same time in parallel. The use of the actual propagator assures that the resultant values are the closest approach.

\section{Performance}

The performance in the sieve mode is dependent on a number of factors. Chief among these are the step size of propagation, the 


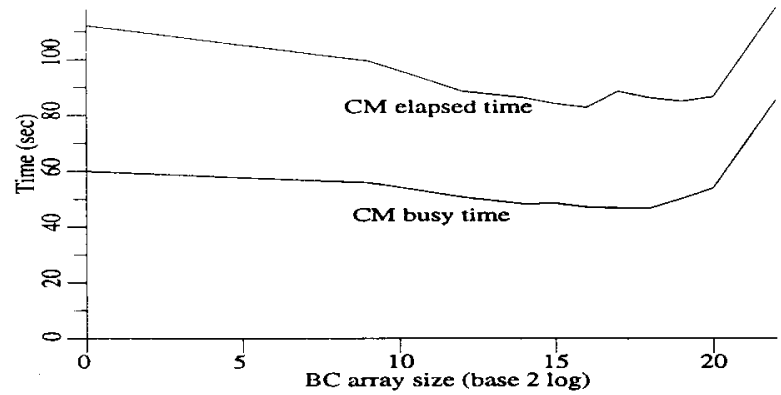

Fig. 2 Time of execution for $1 \mathrm{~h}$ of all-to-all simulation for various $\mathrm{BC}$ array sizes, with step size of $240 \mathrm{~s}$ and $c=2.5 \mathrm{~km}, 256 \mathrm{CM}-5$ nodes.

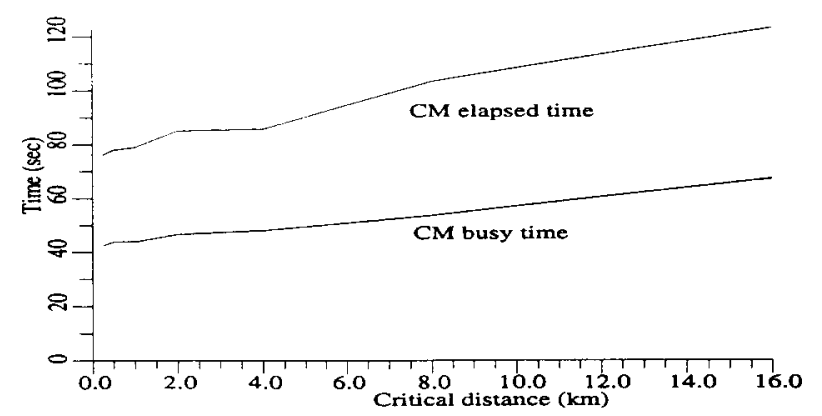

Fig. 3 Time of execution for $1 \mathrm{~h}$ of all-to-all simulation for various critical distances $c$ with step size $240 \mathrm{~s}$ and broadcast size 16, $256 \mathrm{CM}-5$ nodes.

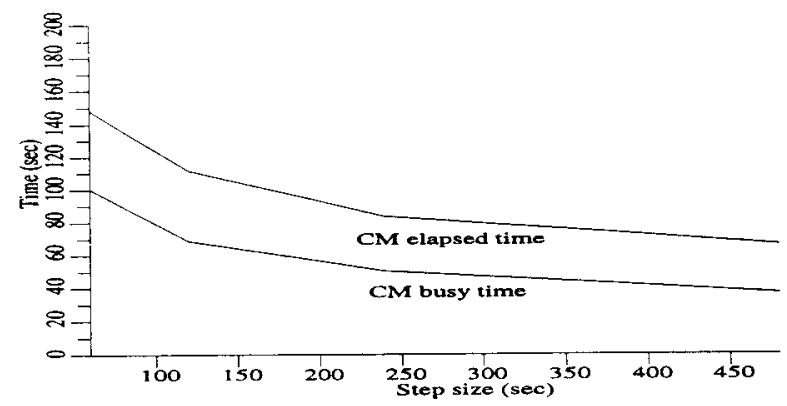

Fig. 4 Time of execution for $1 \mathrm{~h}$ of all-to-all simulation for various step sizes, with $c=1.0 \mathrm{~km}$ and $\mathrm{BC}$ size $16,256 \mathrm{CM}-5$ nodes.

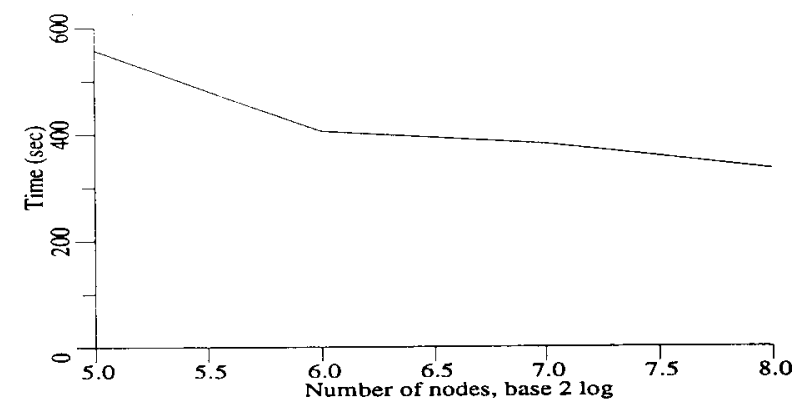

Fig. 5 Time of execution for $30 \mathrm{~min}$ of all-to-all simulation, 3-min step size, with $c=2.5 \mathrm{~km}$, on different-size machines.

critical distance $c$, and the BC array size. Also, the number of processors being used is obviously important, as is the size of the satellite dimension for the arrays. The figures below show the relative performance of a 1-h all-to-all close conjunction detection on the catalog of 7613 satellites from May 24, 1993. The figures have both CM elapsed time and CM busy time; the gap between the two times indicates a significant amount of nonparallel work performed on the process manager; as the number of satellites increases, we can expect greater parallelization and a relative closing of the gap

shows the dependence on the size of the BC arrays, Fig. 3 shows the dependence on critical distance $c$, and Fig. 4 shows the dependence on the time step. Although Fig. 4 shows that a larger step size is quicker, care must be taken. In this particular hour, there were two coincidental conjunctions, one at a distance of $0.970 \mathrm{~km}$ and the other at a distance of $0.994 \mathrm{~km}$. With $c$ set to $1 \mathrm{~km}$, both were missed by the simulation with step size $480 \mathrm{~s}$ and the higher one was missed with a step size of $240 \mathrm{~s}$. This is because the actual distance is so close to the cutoff that the linear extrapolation misses the conjunction for longer step size. One may compensate for this by setting the cutoff slightly higher; for the 240 -s step simulation, the program reported that the maximum of the distance adjustments was 0.094 $\mathrm{km}$. Therefore, allowing for estimation errors of about double this (giving a safety factor) should capture both conjunctions; indeed, for $c=1.2 \mathrm{~km}$, both appear. Finally, Fig. 5 shows the dependence of execution time on a CM-5 with the $\log _{2}$ number of nodes. A CM-5 node consists of a SPARC processor and four vector units. Of course, although an increased number of nodes decreases execution time, it is not "cost effective" for a fixed number of satellites; that is, the product of time and number of nodes increases with an increase in the number of nodes.

\section{New Studies}

The unique capability of this program is to find close conjunctions in an all-to-all comparison of a large set of satellites against itself. By using the actual Space Surveillance catalog and running a simulation

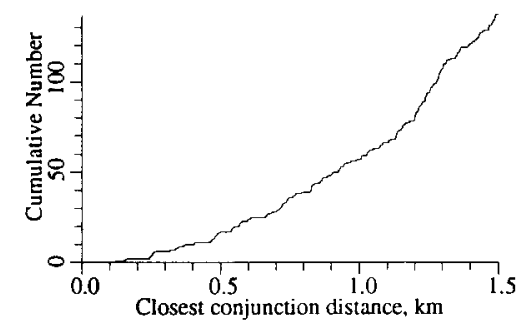

Fig. 6 Number of close conjunctions in 24-h period less than or equal to given distance.

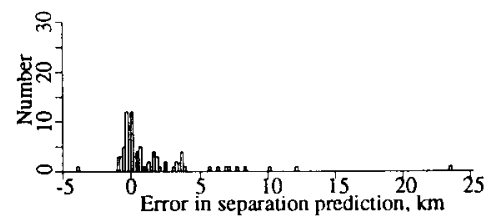

a) Four days

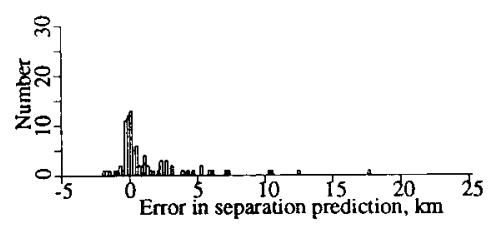

b) Three days

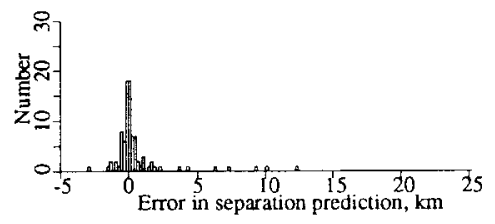

c) Two days

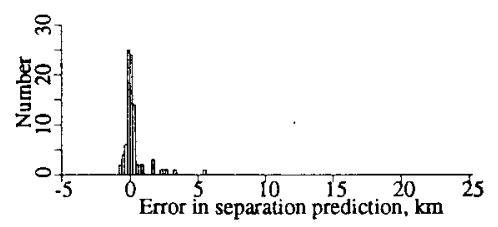

d) One day

Fig. 7 Histogram of errors in conjunction distance for catalogs of various ages prior to conjunction date. 
Table 2 Satellites broken up on July 27, 1993, 00:00 in simulation

\begin{tabular}{|c|c|c|c|c|c|c|}
\hline $\begin{array}{l}\text { Identification } \\
\text { number }\end{array}$ & $\begin{array}{l}\text { Semimajor } \\
\text { axis, km }\end{array}$ & Eccentricity & $\begin{array}{c}\text { Inclination, } \\
\text { deg }\end{array}$ & $\begin{array}{c}\text { Mean anomaly, } \\
\text { deg }\end{array}$ & $\begin{array}{c}\text { Argument perigee, } \\
\text { deg }\end{array}$ & $\begin{array}{l}\text { Right ascension of } \\
\text { ascending node, deg }\end{array}$ \\
\hline 118 & 7280.58 & 0.009158 & 66.78 & -24.3 & 49.2 & 159.5 \\
\hline 211 & 7101.36 & 0.010261 & 66.58 & -158.4 & 166.1 & 56.2 \\
\hline 4724 & 7431.89 & 0.065031 & 62.81 & 51.1 & -105.9 & 133.4 \\
\hline 11671 & 6962.73 & 0.003230 & 82.51 & 172.3 & -306.5 & 61.4 \\
\hline 11694 & 7847.57 & 0.001765 & 74.03 & -2.2 & -12.1 & 11.0 \\
\hline 14801 & 7146.72 & 0.000728 & 74.07 & 155.0 & -208.3 & 83.2 \\
\hline 14814 & 7072.02 & 0.001526 & 74.03 & 11.6 & 154.2 & -44.4 \\
\hline 17911 & 7006.59 & 0.002355 & 82.51 & -128.7 & 134.4 & 8.8 \\
\hline 19396 & 7053.58 & 0.005222 & 58.50 & -96.8 & 95.6 & -152.5 \\
\hline 19440 & 7730.94 & 0.018462 & 73.75 & 178.2 & -262.1 & 127.8 \\
\hline
\end{tabular}

Table 3 Close conjunctions within $1 \mathrm{~km}$ for simulated breakup (July 2, 1993)

\begin{tabular}{rccc}
\hline \hline $\begin{array}{l}\text { Identification } \\
\text { number 1 }\end{array}$ & $\begin{array}{c}\text { Identification } \\
\text { number 2 }\end{array}$ & $\mathrm{km}$ & Time \\
\hline 4578 & 1011270 & 0.329 & $17: 53: 40.308$ \\
12347 & 1009174 & 0.354 & $17: 50: 56.164$ \\
5266 & 1008571 & 0.481 & $15: 41: 59.240$ \\
20029 & 1008917 & 0.504 & $13: 07: 17.362$ \\
86654 & 1009569 & 0.649 & $15: 07: 29.632$ \\
5350 & 1008548 & 0.712 & $13: 58: 09.069$ \\
87590 & 1008679 & 0.808 & $16: 23: 54.327$ \\
15472 & 1009382 & 0.955 & $13: 47: 42.779$ \\
9617 & 1009301 & 0.976 & $16: 51: 23.967$ \\
17192 & 1009065 & 0.994 & $17: 49: 32.322$ \\
\hline \hline
\end{tabular}

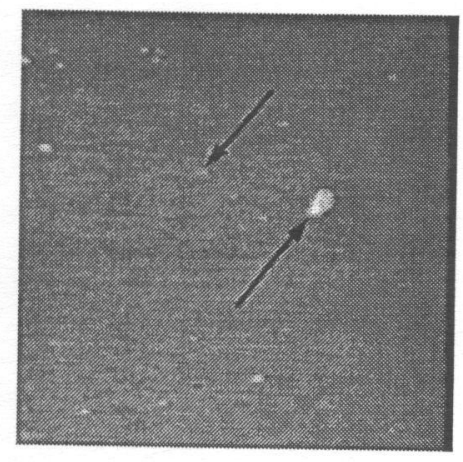

Fig. 8 Photograph of TIROS 10 (upper arrow) and NOAA 10 (lower arrow), on May 21, 1993.

for $24 \mathrm{~h}$, one can assess the degree of crowding in the space environment. Using the catalog starting January 17, 1993, at 14:00:00, we found all satellites that came within $2.0 \mathrm{~km}$ of another satellite in the catalog. In this period, there were 140 coincidental conjunctions. There were 57 that came within $1 \mathrm{~km}$; Fig. 6 shows the cumulative number of conjunctions for each distance. The work of Khutorovsky et al., ${ }^{7}$ taking a different approach, supports these results.

As a measure of the effectiveness of long-term prediction with the existing propagator PPT $2^{8}$ one can predict the conjunctions several days off and then, with daily catalogs updated with new observations, try to predict again for that time period. As the actual date gets closer, the conjunction distance should converge to the actual value. Figure 7 shows the distribution of errors made in predicting the closest conjunction distance with catalogs $4,3,2$, and 1 day prior to the day of conjunction.

As a dramatic indication of a conjunction, the Naval Space Command ran conjunction predictions daily of 177 satellites with active payloads against the catalog using $\mathrm{CM}-\mathrm{COMBO}$. A conjunction was predicted for TIROS 10, launched July 2, 1965, and NOAA 10 , launched September 17, 1986 (two weather satellites). The closest distance predicted was $2.113 \mathrm{~km}$ on May 21, 1993, 05:53:27.876. This was videotaped by the Air Force Maui Optical Station in Hawaii, and a frame from that videotape showing the conjunction is provided in Fig. 8.
One simulation that can be performed easily with this program is the computation of conjunctions of the existing catalog to a debris cloud. With this CM program and the actual catalog from the Naval Space Command for July 27, 1993, we simulated the explosions of 10 low-Earth-orbit satellites, each into 400 fragments, with peak distribution speeds of $100 \mathrm{~m} / \mathrm{s}$. A simulation with 4-min time steps for a period of $6 \mathrm{~h}$ after the explosions comparing the debris against the actual remaining catalog showed that 297 different satellites from the catalog would come within $5.0 \mathrm{~km}$ of a fragment, with the closest approach being $0.329 \mathrm{~km}$. The run time on the 256 -node CM-5 for this simulation was about $17.5 \mathrm{~min}$. The satellites whose breakup was simulated, with their orbital elements, are given in Table 2, and the conjunctions within $1 \mathrm{~km}$ are given in Table 3.

\section{Conclusions}

Using a parallel computer, it is possible in a reasonable amount of time to find and classify the conjunctions all to all within a large ( 8000 or more satellite) catalog or between two large catalogs. This is accomplished with a sieve, which by sorting the Cartesian positions is able to eliminate from consideration the vast majority of possible pairs. This method has been implemented on a CM and was used to predict a conjunction of two weather satellites (which were then subsequently photographed) and has been used for several debris studies.

\section{Acknowledgments}

This work was supported by the Naval Space Command. Bernie Kelm of Allied Signal Technical Services was instrumental in the development and operation of this program. Edna Jenkins and Jon Boers of the Naval Space Command were very helpful in performing the studies described in Sec. VII. Shannon Coffey of the Naval Research Laboratory and Steve Knowles of Naval Space Command were essential in bringing this project about.

\section{References}

${ }^{1}$ Hoots, F. R., Crawford, L. L., and Roehrich, R. L., "An Analytic Method to Determine Future Close Approaches Between Satellites," Celestial Mechanics, Vol. 33, 1984, pp. 143-158.

${ }^{2}$ Mietz, L. A., "Predicting Line-of-Sight Conjunction Between a Specific Satellite and Others," M.S. Thesis, Air Force Inst. of Technology, 1973; also document AD-774388, available from National Technical Information Service, Springfield, VA.

${ }^{3}$ Alfano, S., and Negron, D., "Determining Satellite Close approaches," Journal of the Astronautical Sciences, Vol. 41, 1993, pp. 217-226.

${ }^{4}$ Dybczyński, P. A., Jopek, T. J., and Serafin, R. A., "On the Minimum Distance Between Two Keplerian Orbits with a Common Focus," Celestial Mechanics, Vol. 38, 1986, pp. 345-356.

${ }^{5}$ Beerer, J., and Bauer, T., "Determination of Closest Approach and Duration of Encounter for Two Satellites in Circular Non-Coplanar Orbits," Advances in the Astronautical Sciences, Vol. 54, 1983, pp. 335-343; also American Astronautical Society, Paper AAS 83-336.

${ }^{6}$ Phipps, W. E., Neta, B., and Danielson, D. A., "Parallelization of the Naval Space Surveillance Satellite Motion Model," Journal of the Astronautical Sciences, Vol. 41, 1993, pp. 207-216.

${ }^{7}$ Khutorovsky, Z., Boikov, V., Kamensky, S., Makhonin, G., and Smelow, V., "Collision Risk Analysis for Satellites in LEO," NAVSPACOM/Russia Orbit Determination and Prediction Workshop Washington, DC, July 1994.

${ }^{8}$ Solomon, D., "The NAVSPASUR Satellite Motion Model," Naval Research Lab., Washington, DC, 1991. 